\title{
Comparative Study of Dielectric Properties and Relative Magnetic Permeability of Nano and Bulk Mg Zn Spinel Ferrite
}

\author{
D. El Kony and S. A. Saafan \\ Department, Faculty of Science, Tanta University, Tanta 31527, Egypt
}

\begin{abstract}
Mixed ferrite of composition $\mathrm{Mg}_{0.8} \mathrm{Zn}_{0.2} \mathrm{Fe}_{2} \mathrm{O}_{4}$ was prepared by two different methods, the standard double sintering ceramic technique was used for bulk sample preparation. Nano particle sample was prepared by wet chemical method. The nano-sample was calcinated at different temperatures in order to obtain different particle sizes for performing a comparative study. $X$-ray diffraction using $\mathrm{Cu} \mathrm{k}_{\alpha}$ radiation confirmed the formation of the required ferrites. A.C. conductivity, dielectric function $(\varepsilon)$ and the dielectric loss tangent (tan $\delta)$ were measured as functions of frequency and temperature, the results are reported in the present paper and it was found that the studied properties are influenced by the preparation method and the sintering temperature.
\end{abstract}

\section{Introduction}

Soft ferrites are employed in a wide range of applications and had contributed historically to advances in electronics [1]. Mg ferrite is known to be a good candidate in (lower requirement) television yokes and fly-back transformers because of the lower cost of $\mathrm{Mg}$ and its higher resistivity that eliminates the need for taped insulation between yoke and winding. $\mathrm{Mg}$ ferrite may be used in conjunction with other divalent ions (such as $\mathrm{Zn}$ ) in higher frequency applications for resistivity reasons. [2]. Many studies of related mixed ferrites show interesting properties of these compounds [3-5]. By introducing nonmagnetic ions such as $\mathrm{Zn}^{2+}$ in $\mathrm{Mg}$-ferrite a very large influence on its magnetic properties is observed. A considerable effect on other properties of magnesium ferrite such as dielectric behavior, electrical resistivity, etc. are also studied [6].

Nowadays, Magnetic nanoparticles have been drawing much attention because of their unique features, such that the nano-magnetic particles may have properties extremely different from their bulk counterparts due to the large surface to bulk ratio. This can lead to spin canting, surface anisotropy, 
superparamagnetism (SP) and / or dislocations, etc. This makes them quite flexible to tailor the material for specific applications [7].

Every day $\mathrm{Mg}$ ferrite prove growing importance for technological applications, not only in high-density magnetic recording systems and sensors but also as promising materials in the medical field [8].

However, dielectric and /or transport properties of ferrites such as dielectric constant, ac conductivity and loss tangent provide information suitable for the selection of these materials for specific applications and give valuable information about conduction mechanism. These properties depend on the method of preparation, types of additives, temperature and sintering conditions [9].

The aim of the present work is to perform a comparative study of the dielectric properties of nano-samples and their bulk counterpart of the mixed ferrite $\left(\mathrm{Mg}_{0.8} \mathrm{Zn}_{0.2} \mathrm{Fe}_{2} \mathrm{O}_{4}\right)$. The nano samples were prepared by the co-precipitation method and sintered at different temperatures in order to obtain three samples with different average particle size while the bulk sample was prepared by the standard ceramic technique.

\section{Experimental}

A bulk sample (S1) of the mixed ferrite $\left(\mathrm{Mg}_{0.8} \mathrm{Zn}_{0.2} \mathrm{Fe}_{2} \mathrm{O}_{4}\right)$ was prepared by the standard double sintering ceramic technique and three nano samples of the same composition were prepared by the co-precipitation method. The sample (S2) is the as prepared, (S3) was calcinated at $873 \mathrm{~K}$ for $5 \mathrm{~h}$ and (S4) was calcinated at $1523 \mathrm{~K}$ for $5 \mathrm{~h}$. X-ray diffraction by using an X-ray diffractometer (PRUKER-DX) was performed to check the formation of the required ferrite.

A.C. conductivity, dielectric constant, and loss tangent $\tan \delta$ are studied as functions of frequency at different temperatures by using the circuit shown in reference [10]. The samples have been pressed into pellets. The surfaces of each disc-shaped sample have been coated by silver paste to improve the contact with the two electrodes placed in an evacuated silica tube surrounded by a home-made heater. In this circuit, the sample is connected in series with a resistor $R$ that must be adjusted such that the value of $V_{R}$ is small enough ( $<$ 0.1 ) compared to the applied voltage, V, (r.m.s $=1$ Volt). The lock-in amplifier (Stanford Research Systems SR 510) reads the voltage drop, $V_{R}$, on the resistor, $R$, and the phase angle, $\phi$, between the applied voltage, $V$, and the current I passing through the resistor $R$. The sample can be considered as a real capacitor; which can be represented by an ideal capacitor " $C$ " shunted with a resistance " $r$ '. The current through the sample consists of two parts, the 
displacement current, $\mathrm{I}_{\mathrm{C}}$, and the conduction current $I_{r}$ which lags behind $I_{C}$ by an angle $\pi / 2 . \quad V_{R}$ and $\phi$ were measured at different temperatures and frequencies for all samples. AC conductivity $\sigma_{\mathrm{ac}}$, real dielectric constant $\varepsilon^{\prime}$ and dielectric loss $\tan \delta$ were calculated as functions of frequency at different temperatures by using the following equations:

$$
\begin{aligned}
& \sigma_{a c}^{\prime}=\frac{d}{r A}=\frac{d V_{R} \cos \phi}{A V R} \\
& \varepsilon^{\prime}=\frac{\boldsymbol{C}}{\boldsymbol{C}_{\boldsymbol{o}}}=\frac{11.3 \boldsymbol{d} \boldsymbol{V}_{\boldsymbol{R}} \sin \phi}{\boldsymbol{R A} \omega} \times 10^{12}
\end{aligned}
$$

where $d$ is the thickness of the sample $(\mathrm{cm}), A$ is its surface area $\left(\mathrm{cm}^{2}\right)$ and $\omega=2 \pi f$

$$
\tan \delta=\frac{1}{\tan \phi}=\frac{I_{r}}{I_{C}}
$$

Moreover, the relative magnetic permeability has been measured by a resonance circuit as a function of temperature $[11,12]$.

\section{Results and discussion}

\subsection{X- Ray diffraction patterns}

$\mathrm{X}$-ray diffraction patterns of the mixed ferrite $\left(\mathrm{Mg}_{0.8} \mathrm{Zn}_{0.2} \mathrm{Fe}_{2} \mathrm{O}_{4}\right)$ are presented in Fig. (1). The matching of the standard d-values with the experimentally calculated ones from the diffraction patterns confirms the formation of a cubic spinel structure.

\subsection{AC conductivity behavior}

\section{(a) AC conductivity as a function of frequency:}

Figure (2: a-d) shows the ac conductivity $\sigma_{\text {ac }}^{\prime}$ behavior as a function of frequency at different temperatures. It is known that the complex ac conductivity is given by

$$
\sigma^{*}(\omega)=\sigma^{\prime}+i \sigma^{\prime \prime}
$$

Where, the real part of this function consists of two components:

$$
\sigma^{\prime}=\sigma_{1}(T)+\sigma_{2}(T, \omega)
$$



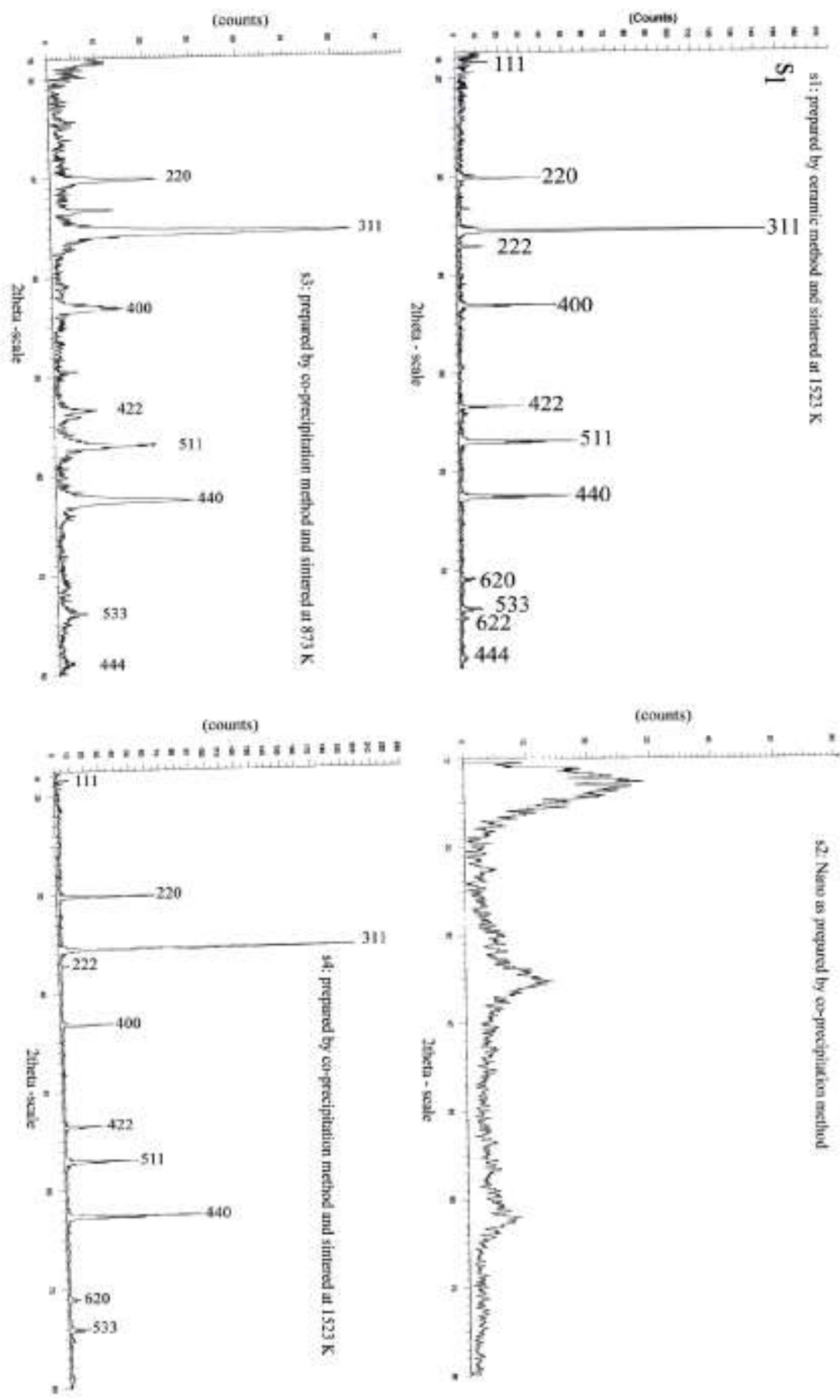

Fig. (1: a-d): shows the X-ray diffraction patterns of the four investigated samples. 


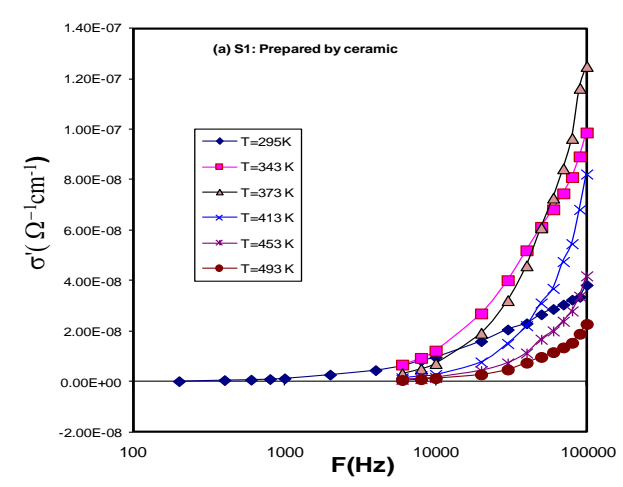

Fig. $(2$ - a)

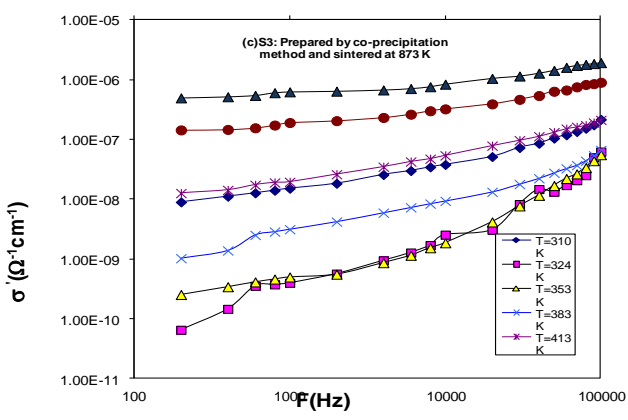

Fig. $(2$ - c)

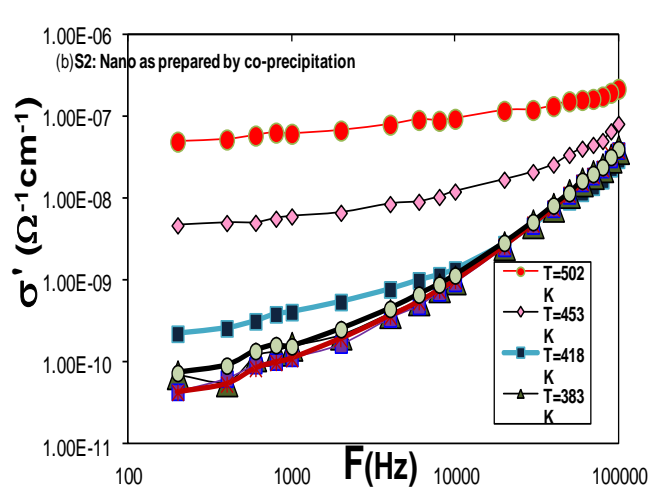

Fig. $(2$ - b)

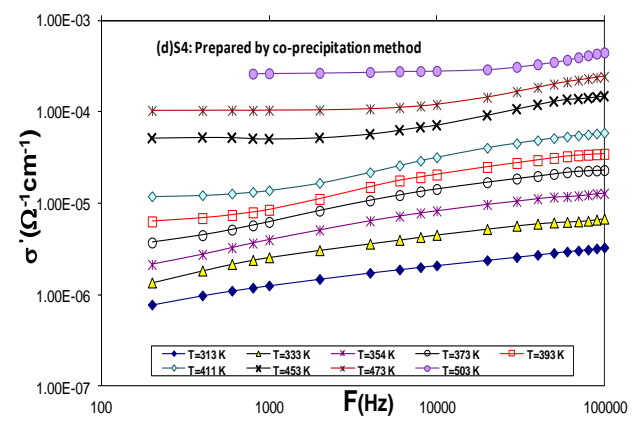

Fig. $(2$ - d)

Fig. (2: a-d): shows The variation of ac conductivity with frequency at different temperatures

The first term, $\sigma_{l}(T)$ is actually the dc electrical conductivity $\sigma_{d c}(T)$, it is temperature dependent. It is related to the drift mobility of electric charge carriers and obeys an Arrhenius relation [11]. The second term $\sigma_{2}(T, \omega)$ is frequency and temperature dependent, it is attributed to the dielectric relaxation caused by the localized electric charge carriers and obeys the power law form:

$$
\sigma_{2}(T, \omega)=B \omega^{s}
$$

Where, $B$ and $s$ are composition and temperature dependent parameters [13, 14].

Figure (2: a-d) shows that the A.C. electrical conductivity $\sigma^{\prime}$ increases with increasing frequency as expected. Different authors [15,16,17], have considered ferrite samples as to be formed of different regions with different 
conductivities and interpreted the similar results by considering space charge polarization and Koop's two-layer model [18]. It is suitable to describe heterogeneous structures; where the ferrite is assumed to be made up of well conducting grains separated by poor conducting layers or grain boundaries, and conduction takes place by hopping mechanism. The electrons reaching the grain boundary through hopping will pile up there due to its higher resistivity, thereby producing space charge polarization. The resistive grain boundaries were found to be more effective at lower frequencies while the conductive ferrite grains are more effective at higher frequencies $[13,14]$

\section{Temperature dependence of the A.C. conductivity:}

Over a limited range of temperature from about $300 \mathrm{~K}$ up to $500 \mathrm{~K}$, because the Curie temperature is expected to be around 400K[19], the A.C. conductivity was measured at different frequencies for the investigated composition as shown in Figure (2: a-d). It can be seen that the $\mathrm{Mg}-\mathrm{Zn}$ ferrite shows generally a semiconducting behavior, as commonly seen in most ferrites. Exceptions in a few temperature values have been observed and may be attributed to variations in the cation distribution as the temperature changes. It is well known that $\mathrm{MgFe}_{2} \mathrm{O}_{4}$ has been reported to have a random cation distribution [20] where a fraction of the $\mathrm{Mg}^{2+}$ ions displace an equivalent number of $\mathrm{Fe}^{3+}$ from octahedral sites (B) where $\mathrm{Mg}^{2+}$ ions have a strong preference to occupy $\mathrm{B}$ sites and partially occupy A sites. By introducing $\mathrm{Zn}^{2+}$ ions in the composition more $\mathrm{Fe}^{3+}$ ions will be displaced to the $\mathrm{B}$ - sites because $\mathrm{Zn}$ has an A-site preference [6]. The conditions of the preparation play an important role in site preference of the cations and consequently in all other properties of the spinel ferrites [1]. Since stability in behavior is important in practical applications, therefore from the present experimental observations it can be concluded that the better method to prepare the investigated composition is that of sample S4 i.e. co-precipitation method then calcinating the precipitates at $1523 \mathrm{~K}$ for five $\mathrm{h}$. This may be due to the fact that the instability due to cationic redistributions seems to disappear in this sample.

\subsection{The dielectric constant behavior}

The dependence of the dielectric constant, $\varepsilon^{\prime}$, on frequency at different temperatures is shown in Fig. (3:a-d). The values of $\varepsilon^{\prime}$ are observed to be diverse ranging from lower values in S1 and S2 and higher values in S3 and S4, this diversity in values is observed in literature $[6,14,21]$. This can be accounted for by variation in microstructure and cationic distribution due to the method and conditions of preparation. The dielectric constant measured at different temperatures is found to decrease as the frequency increases. It can be 
seen that this decrease is more observed at lower frequencies than at higher frequencies. It is pertinent to mention that the high values of dielectric constant of samples S3 and S4 may be attributed mainly to the basis of Koops phenomenological theory [17]. That is, in the case of polycrystalline ferrite, the system can be thought of as consisting of well-conducting grains (with resistivity $\rho_{1}$, dielectric constant $\varepsilon_{1}$, thickness $d_{1}$ ) separated by highly resistive grain boundaries (with $\rho_{2}, \varepsilon_{2}, \mathrm{~d}_{2}$ ) [22].

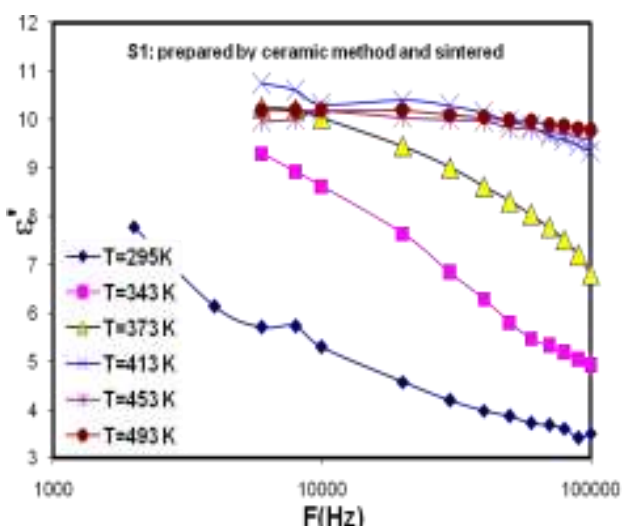

Fig. (3 - a)

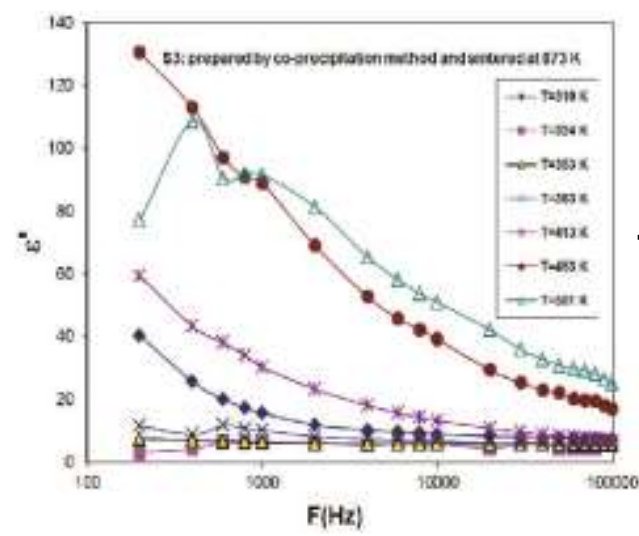

Fig. $(3$ - c)

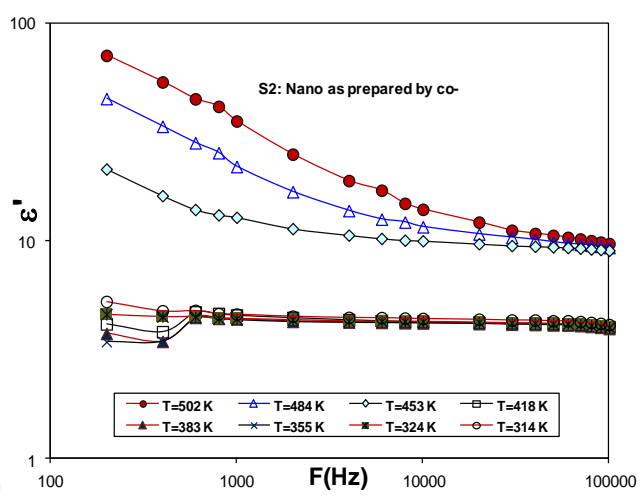

Fig. $(3$ - b)

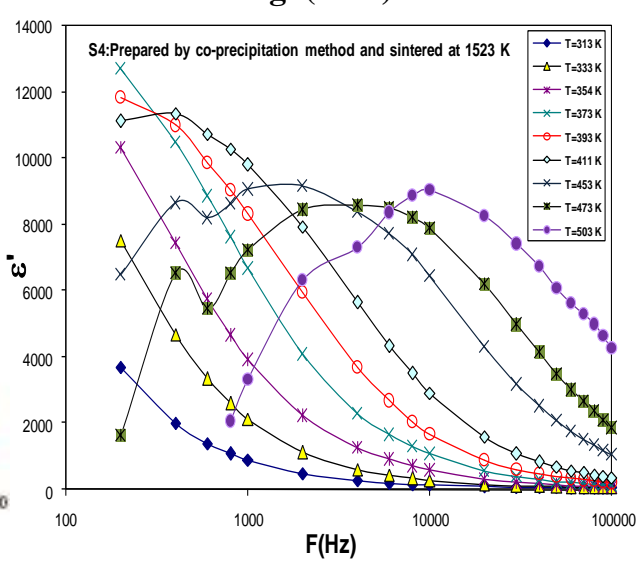

Fig. $(3$ - d)

Fig. (3: a-d): shows The variation of dielectric constant with frequency at different temperatures

The charge carriers reach the grain boundary by hopping encounter different resistances so that accumulation of charges at the separating boundaries occurs causing what is called interfacial polarization and hence the dielectric constant value is highly raised $[15,16,17]$. The behaviour of the 
dielectric constant at lower frequency is mainly controlled by the grain boundary phase [18]. It is worth mentioning that the hopping electron causes local displacement in the direction of the external applied field increasing the dielectric polarization [13, 14]. Therefore, there is a strong correlation between the conduction mechanism and the dielectric behavior of the ferrites starting with the assumption that the mechanism of the polarization process in ferrites is similar to that of the conduction process [21].

However, as the frequency of the applied field is increased, the dielectric constant decreases until reaching a constant value. Since beyond a certain frequency of the applied electric field the polarization cannot follow its changes [23].

Concerning again the variation of the dielectric constant with temperature an increase in the value of dielectric constant is observed with increasing temperature, the increase being quite significant at lower frequencies. The increase in temperature thermally activates the charge carriers, increasing the electron exchange interactions, thereby enhancing the dielectric constant [15]. It is known that there are four main contributions to the polarization in ferrites viz. electronic, atomic, dipolar and interfacial polarization. Electronic and atomic polarization are important at high frequencies and are temperature independent, while the latter two play dominant role at lower frequencies and they are both temperature dependent. Also, interfacial polarization is known to increase with temperature whereas dipolar polarization decreases with temperature $[10]$. The observed increase in dielectric constant with temperature at low frequency is supportive of the fact that interfacial polarization is the main contributor to the dielectric constant in the present samples.

In addition it can be seen that the value of $\varepsilon^{\prime}$ of the nano-sample heated to $1523 \mathrm{~K}$ i.e. $\mathrm{S} 4$ is the highest compared to the other samples. And at some high temperatures it is found an unusual behavior for the dielectric constant as a function of frequency that is; at first the dielectric constant increases to a maximum with increasing frequency, then the usual behaviour (relaxation of the direction constant) occurs with further increase of frequency. This is attributed - according to N. Rezlescu and E. Rezlescu [24] - to the existence of p- type charge carriers, therefore, a collective contribution of the two types of carriers, $p$ and n, makes up the polarization. Again, the sample S4 seems to be the more useful one amongst the samples since the ferrites having very high dielectric constants are useful in designing good microwave devices such as isolators, circulators[21]. 


\subsection{The dielectric loss behavior}

The variation of dielectric loss tangent $(\tan \delta)$ as a function of frequency $(f)$ at different temperatures for the investigated samples is shown in Fig. (4:a-d). The dielectric loss behavior is in general agreement with previous data $[6,13,14,21]$. It decreases with an increase of frequency. Whenever an increase with increasing frequency until reaches a maximum occurs then decreasing again appears, this is attributed to a resonance of the hopping frequency of charge carriers with the frequency of the externally applied electric field [25].

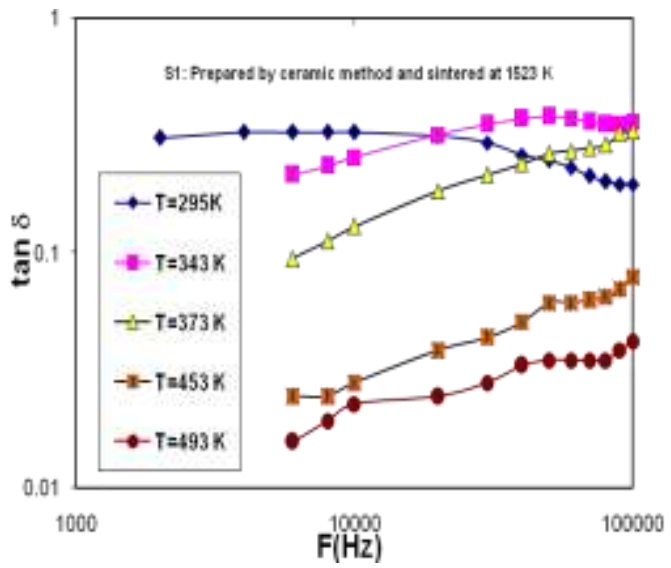

Fig. (4 - a)

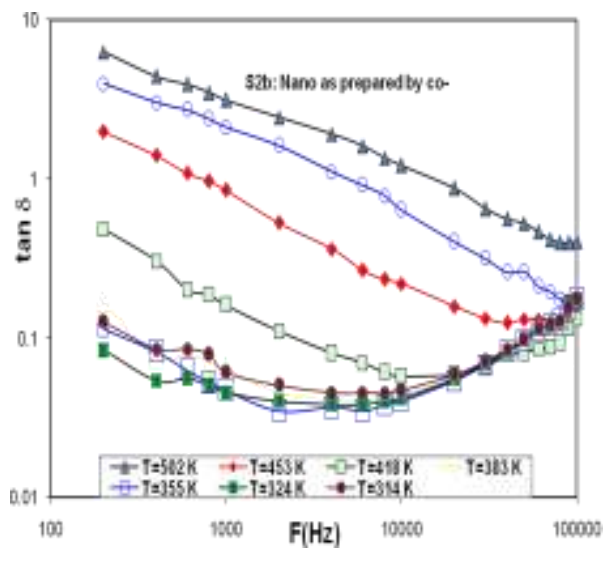

Fig. (4 - b)

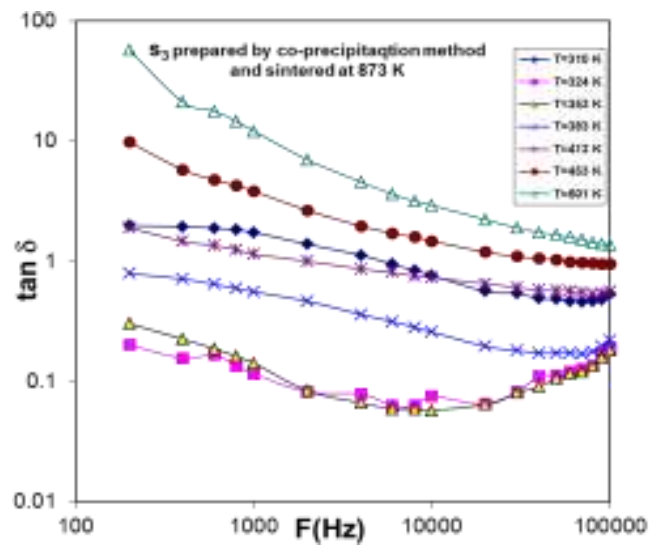

Fig. (3 - c)

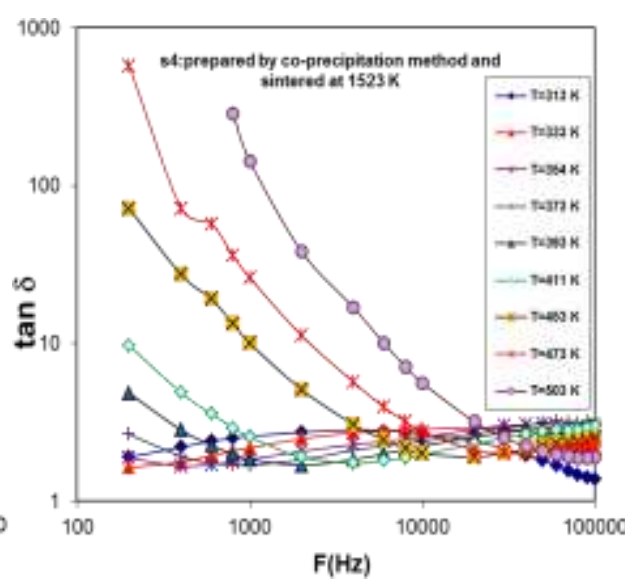

Fig. $(3$ - d) 


\subsection{Relative Magnetic Permeability Measurements}

It is well known that the basic properties of ferrites have been found to be very sensitive to the distribution of metallic ions among the available $\mathrm{A}$ and B sites, chemical composition and upon the particle size, which depends in turn on the method of synthesis [26].

The value of the relative permeability $\mu_{\mathrm{r}}$ gives an indication of how easily a given material can be magnetized compared to free space, so that for free space $\mu_{\mathrm{r}}=1$. Table (1) shows the measured relative permeability, where it is found to be slightly greater than 1 . This is attributed to the fact that $\mu_{\mathrm{r}}$ measurements were performed using powdered samples with much lower density than toroidal shape pressed sintered ferrite samples, and it is known that ferrites with lower density possess a lower permeability. A decrease in the density, not only results in the increase of demagnetizing field due to the presence of pores but also causes the spin rotational contribution to permeability to fall, which in turn decreases the permeability [27]. The values of relative permeability of the as- prepared nano-samples are the lowest as expected according to the literature. This is attributed to random canting of particle surface spins ; where the ratio of surface volume is significant in nanoparticles leading to the reduction in magnetic permeability in ferrite nanoparticles [11].

The results also show a satisfying stability of the relative permeability $\mu_{\mathrm{r}}$ below the Curie temperature in agreement with literature [19]. Again S4 shows the higher values of permeability of the four investigated powder samples.

Table (1): Relative permeability of different samples at different temperatures

\begin{tabular}{|c|c|r|r|c|}
\hline $\mathrm{T}(\mathrm{K})$ & \multicolumn{1}{|l|}{$\mathrm{S} 1: \mu_{\mathrm{r}}$} & \multicolumn{1}{|l|}{ S2: $\mu_{\mathrm{r}}$} & S3: $\mu_{\mathrm{r}}$ & S4: $\mu_{\mathrm{r}}$ \\
\hline 306 & 1.31 & 1.04 & 1.21 & 1.42 \\
\hline 323 & 1.27 & 1.05 & 1.22 & 1.39 \\
\hline 333 & 1.22 & 1.03 & 1.09 & 1.4 \\
\hline 353 & 1.21 & 1.07 & 1.21 & 1.41 \\
\hline 383 & 1.21 & 1.04 & 1.23 & 1.4 \\
\hline 413 & 1.2 & 1.03 & 1.21 & 1.38 \\
\hline 453 & 1.2 & 1.02 & 1.24 & 1.38 \\
\hline 483 & 1.19 & 1.02 & 1.16 & 1.39 \\
\hline
\end{tabular}




\section{Conclusion}

Since stability in behavior is important in practical applications, therefore from the present experimental comparative study it can be concluded that the better composition is sample $\mathrm{S} 4$ prepared by the co-precipitation method then calcinated at $1523 \mathrm{~K}$ for five $\mathrm{h}$ because the instability in the conductivity behavior with temperature due to cationic redistributions seems to disappear in this sample. It has also the highest values of dielectric constant which is useful in designing good microwave devices such as isolators, circulators. Concerning the relative permeability too, the sample S4 has the larger values of permeability amongst the investigated samples.

\section{Acknowledgement}

The authors are indebted to Prof. Dr. M.K. El-Nimr the founder of Materials Science and Electronics Lab in the Physics Department, Faculty of Science, Tanta University, Egypt, for his constant support and fruitful advices.

\section{References}

1. H.M. Zaki, Physica B 404, 3356 (2009).

2. A. Goldman, Modern Ferrite Technology, $2^{\text {nd }}$ Ed, (C) 2006 Springer, Science \& Business Media, Inc.

3. P.K. Roy, J. Bera, J. Magn. Magn. Mater. 298, 38 (2006).

4. S.M. Attia, A.M. Abo El Ata, D. El Kony, J. Magn. Magn. Mater. 270 (2004) 142. [5] S.S. Suryavanshi, R.S. Patil, S.A. Patil, S.R. Sawant, J. Less-Common Met. 168, 169 (1991).

5. S.A. Mazen, H.M. Zaki, S.F. Mansour Inter, J. Pure Appl. Phys. 3, 40 (2007).

6. Kumar, V., Rana, A., Yadav, M.S. and Pant, R.P. 'Size-induced effect on nano-crystalline $\mathrm{CoFe}_{2} \mathrm{O}_{4}$ ', J. Magn. Magn. Mater., Vol. 320, pp. 1729, (2008).

7. Y. Ichiyanagi_, M. Kubota, S. Moritake, Y. Kanazawa, T. Yamada, T. Uehashi, Journal of Magnetism and Magnetic Materials, 310, 2378 (2007).

8. A.K.M. Akther Hossaina, S.T. Mahmuda, M. Sekib, T. Kawaib, H. Tabata, J. Magn. Magn. Mater. 312, 210 (2007).

9. M.A. Elkestawy, Journal of Alloys and Compounds, 492, 616 (2010).

10. M.K. ElNimr, B.M.Moharram, S.A.Saafan, S.T.Assar, J. of Magn. Magn. Mater. 322, 2108 (2010). 
Q Qin Yu, Thomas W.Holmes, and Krishna Naishadham, IEEE Transactions on Electromagnetic Compatibility, vol. 44, No. 1, February (2002).

12. A.M. Abdeen, J. Magn. Magn. Mater. 185, 199 (1998).

13. M.A. El Hiti, J. Magn. Magn. Mater. 164, 187 (1996).

14. K. M. Batoo, S. Kumar, C. G. Lee, Alimuddin, J. Curr. Appl. Phys. 9, 1072 (2009).

15. Soibam, S. Phanjoubam, H.B. Sharma, H.N.K. Sarma, R. Laishram, C. Prakash, Solid State Comm., 148, 399 (2008).

16. A. Thakur, P. Mathur. M. Singh, J. Phy. Chem. Sol., 68, 378 (2007).

17. A. G. Koops, Phys. Rev. 83 (1), 121 (1951).

18. M. ManjurulHaque, M.Huq, M.A.Hakim, Physica B 404, 3915 (2009).

19. N. Okasha, J. Mater Sci, 43, 4192 (2008).

20. D.Ravinder, K. Latha, J. Mater. Lett., 41, 247 (1999).

21. H.-B. Wang, J.-H. Liu, W.-F. Li, J.-B. Wang, L. Wang, L.-J. Song, S.-J. Yuan, F.-S. Li, J. Allo. Comp., 461, 373 (2008).

22. A.A. Zaky and R. Hawley, "Dielectric Solids", New York: Dover Publications Inc, (1970).

23. N. Rezlescu and E. Rezlescu, Phys. Stat. Sol., 23(a), 575 (1974).

24. D.Ravinder, K. V. Kumar, J. Bull. Mater. Sci., 24(5), 505 (2001).

25. Santosh S. Jadhava, Sagar E. Shirsath, B. G. Toksha, S. J. Shukla, K. M. Jadhav, Chinese Journal of Chemical Physics vol. 21 no. 4, 381 (2008).

26. P.K. Roy, J. Bera, Journal of Magnetism and Magnetic Materials, 298, 38 (2006). 